\title{
Steering clear of danger
}

Salpingo oophorectomy - the removal of ovaries and fallopian tubes - has long been considered to be a means of preventing ovarian cancer in women with genetic susceptibility to this disease. But until now, little clinical data has supported the efficacy of this approach. Two recent studies published in the New England Journal of Medicine report that this procedure can decrease the incidence of breast cancer and gynaecological cancers in women carrying $B R C A$ mutations.

The BRCA1 and BRCA2 genes encode proteins that participate in the DNA-damage response, and mutations in these genes have been associated with increased susceptibility to breast and ovarian cancers. These

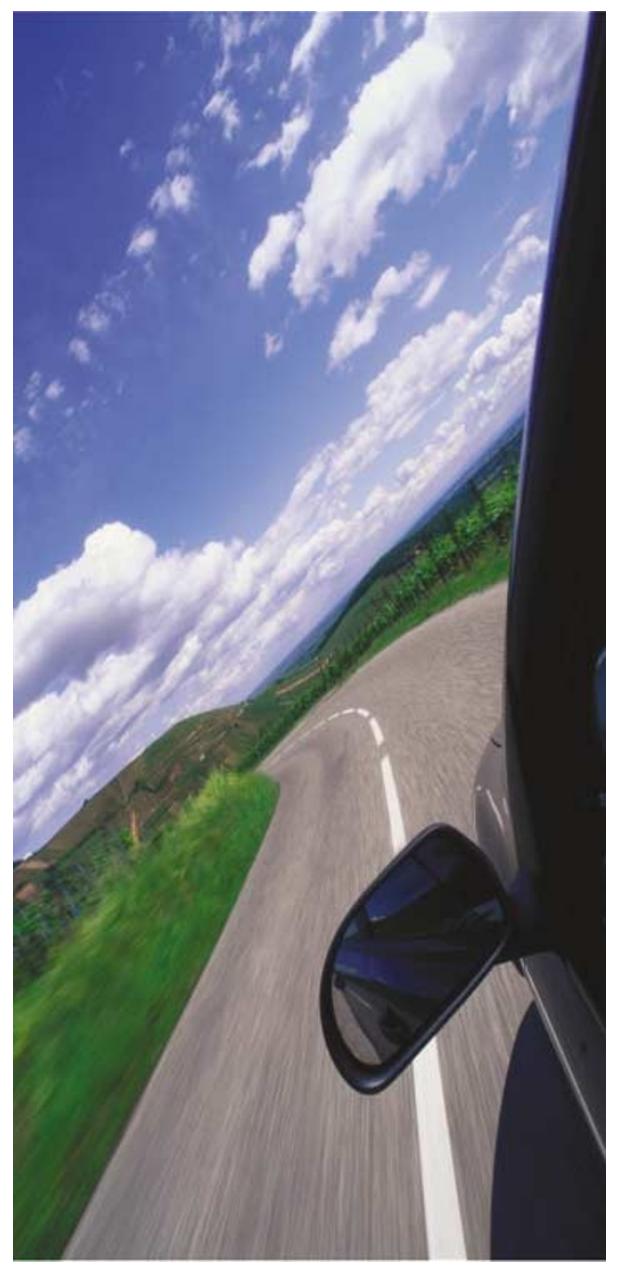

mutations contribute to a small fraction of breast cancer cases, but make up $10 \%$ of cases diagnosed in women under 40 and almost $75 \%$ of all familial cases. Furthermore, about $10 \%$ of all ovarian cancer cases are associated with $B R C A$ mutations. As genetic tests for $B R C A$ mutations are available, preventative measures are needed for patients that test positive for cancerassociated mutations. Carriers of BRCA1 mutations have a $50-85 \%$ lifetime risk of developing breast cancer and a $20-40 \%$ lifetime risk of developing ovarian cancer, whereas women with BRCA2 mutations have a 10-20\% risk of developing ovarian cancer.

Bilateral prophylactic mastectomy has been shown to prevent cancer in these women, but concern about the fact that breast cancer does not develop in all carriers, and the knowledge that early cancers can be treated effectively, have made this a controversial preventative approach. Although risk of ovarian cancer in carriers of BRCA mutations is lower than the risk of breast cancer, the absence of reliable methods for early detection and the high mortality rates for advanced ovarian cancer indicate that prophylactic oophorectomy might be a good option. Fortunately, because ovarian cancer is not usually diagnosed until women are in their 50 s, prophylactic oophorectomy can be delayed until after a woman's child-bearing years are completed.

Data from two large clinical studies now support this theory. Noah Kauff et al. reported the results of a prospective study of $170 \mathrm{BRCA}$ mutation carriers. Of 72 women who elected for intensive surveillance, $6.9 \%$ developed ovarian cancer or papillary serous carcinoma of the peritoneum. Of the 98 women who underwent prophylactic salpingo oophorectomy, however, three had early-stage tumours that were diagnosed at the time of surgery, and primary peritoneal cancer developed in one patient during follow-up (1\%). Breast cancer developed in $12.9 \%$ of women in the surveillance group, but only in $4.3 \%$ of women in the oophorectomy group.

In an accompanying article, Tim Rebbeck et al. reported the results of a multicentre retrospective analysis of 551 BRCA-mutation carriers. Ovarian cancer developed in $20 \%$ of women who chose to undergo surveillance. In women that chose the surgery, stage I ovarian tumours were identified at the time of surgery in six women $(2.3 \%)$, and primary peritoneal cancer only developed in two others $(0.8 \%)$. During 11 years of follow-up, breast cancer developed in $42.3 \%$ of women who underwent surveillance, but only $21.2 \%$ of women who had undergone prophylactic oophorectomy.

Why does oophorectomy prevent breast cancer? The ovaries produce oestrogen, and oestrogen-receptor signalling promotes proliferation of breast tumour cells. Not all BRCAassociated tumours express high levels of the oestrogen receptor, however, so the protective effects of oestrogen antagonists in BRCAmutation carriers have been debated.

The findings of Kauff and Rebbeck indicate that hormonal manipulation does indeed have a preventative effect on $B R C A$-associated cancer. The preventative effect did not, however, require a complete oestrogen blockade, as many of the women in the study received hormone-replacement therapy. But the ability to prevent breast and ovarian cancer in $B R C A$-mutation carriers will encourage genetic testing of women with a family history of breast cancer.

Kristine Novak

(2) References and links ORIGINAL RESEARCH PAPERS Kauff, N. D. et al. Risk-reducing salpingo-oophorectomy in women with a BRCA1 or BRCA2 mutation. N. Engl. J. Med. 346, 1609-1615 (2002) | Rebbeck, T. R. et al. Prophylactic oophorectomy in carriers of BRCA1 or BRCA2 mutations. N. Engl. J. Med. 346, 1616-1622 (2002) FURTHER READING

Haber, D. Prophylactic oophorectomy to reduce the risk of ovarian and breast cancer in carriers of BRCA mutations. N. Engl. J. Med. 346,

1660-1662 (2002)
WEB WATCH

Mad about genomics

- http://cgap.nci.nih.gov

In 1996, the National Cancer Institute (NCl) launched an exciting programme - the Cancer Genome Anatomy Project (CGAP). The goal of the CGAP is to determine the gene-expression profiles of normal, pre-cancer and cancer cells, which could lead to improved detection, diagnosis and treatment for the patient.

The CGAP data are freely available, and the CGAP is the largest contributor of sequences to the expressed sequence tag (EST) database. Some of this information is available elsewhere, but the site is well designed, easy to navigate and up-to-date - for example, the SAGEmap was relaunched in May this year.

The home page links through to eight main sections, covering biological and practical aspects methods, tools and reagents - of the project. The biological sections cover chromosomes, tissues and pathways - the pathway maps, from which you can click through to individual gene information, are particularly helpful - and contain genomic data for humans and mice. These include ESTs, geneexpression patterns, singlenucleotide polymorphisms, cluster assemblies and cytogenetic information. Clicking through from the biology sections to the next level takes the user to pages with all of the tools and

libraries of information that a researcher in the field could wish for. For the first-time user, there are clear instructions for use of these tools.

The slide tour and education resource, both accessed from the home page, explain the basic science behind the CGAP, and are excellent for those who are not familiar with genomics. Additions to the site could include links to other genome projects and biographies of project team members, but overall this is a very useful resource.

Ezzie Hutchinson 